\title{
Congenital Intrahepatic Portosystemic Venous Shunt Presenting with Paraparesis as the Initial Symptom
}

\author{
Masataka Torigoe $^{1}$, Keisuke Maeshima ${ }^{2}$ and Yasushi Takeshita ${ }^{3}$
}

\begin{abstract}
An 85-year-old woman was hospitalized with rapidly progressive paraparesis without altered consciousness, although she was not definitively diagnosed. She developed acute drowsiness and disorientation several days later. An intrahepatic portosystemic venous shunt (IPSVS) was observed on enhanced computed tomography, and hyperammonemia suggested leakage of neurotoxins from the shunt as the etiology of the patient's symptoms. Her neurological symptoms and hyperammonemia improved following transcatheter shunt embolization. We diagnosed her with hepatic myelopathy, which is a rare complication of liver cirrhosis and portosystemic venous shunts. Hepatic myelopathy resulting from a congenital IPSVS has not been previously reported. A diagnosis of hepatic myelopathy should be ruled out in diagnostically difficult cases of paraparesis.
\end{abstract}

Key words: intrahepatic portosystemic venous shunt, hepatic myelopathy, hepatic encephalopathy

(Intern Med 52: 2439-2442, 2013)

(DOI: 10.2169/internalmedicine.52.0881)

\section{Introduction}

An intrahepatic portosystemic venous shunt (IPSVS) is a bypass from the portal vein to the hepatic veins that causes hepatic encephalopathy (HE) primarily via the development of hyperammonemia. In many cases, IPSVS is accompanied by a hepatic pathology, such as portal hypertension or a history of abdominal trauma or surgery, that results in shunt formation. IPSVS is thought to be congenital when no such conditions are present. Congenital IPSVS develops during the embryonic period as a vascular anomaly that manifests with the clinical onset of HE. Altered consciousness, irrational behavior and asterixis are the characteristic symptoms of HE. Although rare, spastic paraparesis can be caused by chronic liver diseases that result in hyperammonemia, a condition called hepatic myelopathy. However, to the best of our knowledge, hepatic myelopathy resulting from congenital IPSVS has not been previously reported. We experienced a case of congenital IPSVS associated with hepatic myelopathy that initially manifested as rapidly progressive paraparesis without altered consciousness and was difficult to differentiate from cerebrovascular disease.

\section{Case Report}

An 85-year-old woman was hospitalized with rapidly progressive bilateral weakness of the lower limbs without altered consciousness. She had become unable to stand or walk within a few hours in August 2011. She had been receiving medications for type 2 diabetes mellitus, hypertension and dyslipidemia and had been treated with antiplatelet therapy since she suffered from a transient ischemic attack (TIA) at 80 years of age. Therefore, she was suspected to have a serious neurological problem resulting from a cerebrovascular event and was transferred to our hospital from her local clinic for a further examination.

On admission, the patient exhibited severe systolic hypertension with a blood pressure of 206/69 mmHg. She was alert, oriented and cooperative on a physical examination. Her body temperature was $36.8^{\circ} \mathrm{C}$, her pulse rate was 73 beats/min and her respiratory rate was 12 breaths/min. A neurological examination, including Barre's test and Mingazzini's test, revealed spastic paraparesis with a strength of

${ }^{1}$ Department of Rheumatology, Oita Red Cross Hospital, Japan, ${ }^{2}$ Department of Internal Medicine I, Faculty of Medicine, Oita University, Japan and ${ }^{3}$ Department of Internal Medicine, Tsukumi Central Hospital, Japan

Received for publication April 29, 2013; Accepted for publication June 14, 2013

Correspondence to Dr. Keisuke Maeshima, maeshima@oita-u.ac.jp 
Table. Laboratory Data on Admission

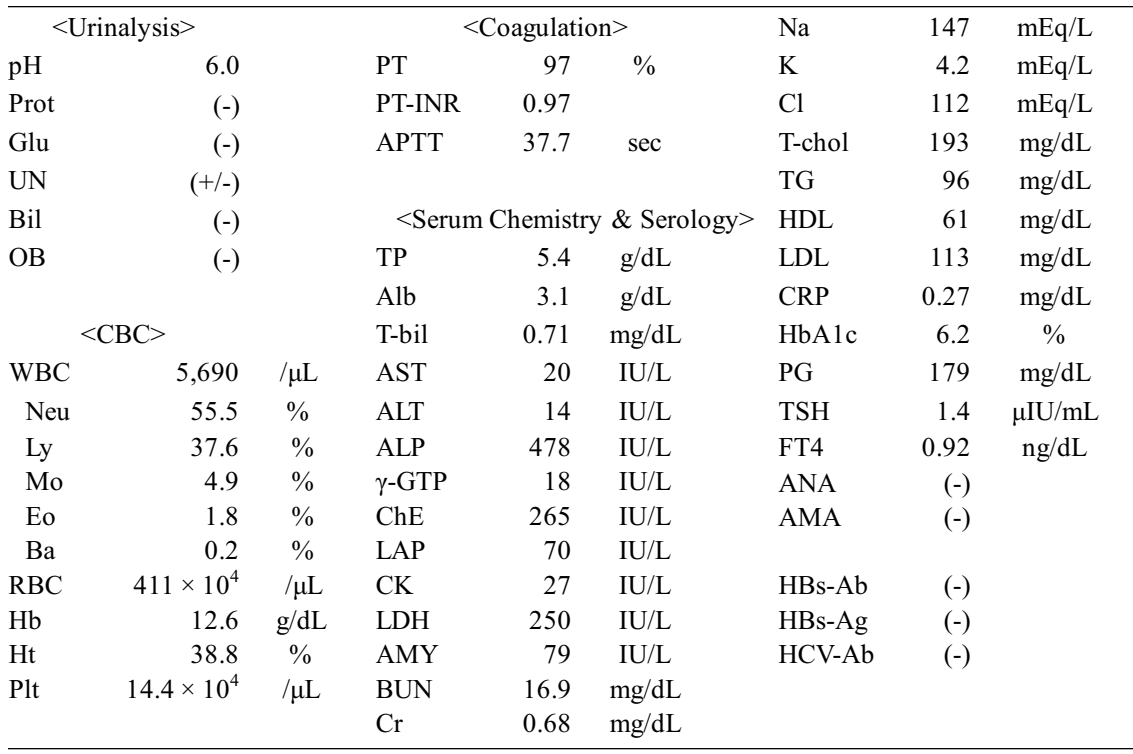

ANA: antinuclear antibody, AMA: antimitochondrial antibody
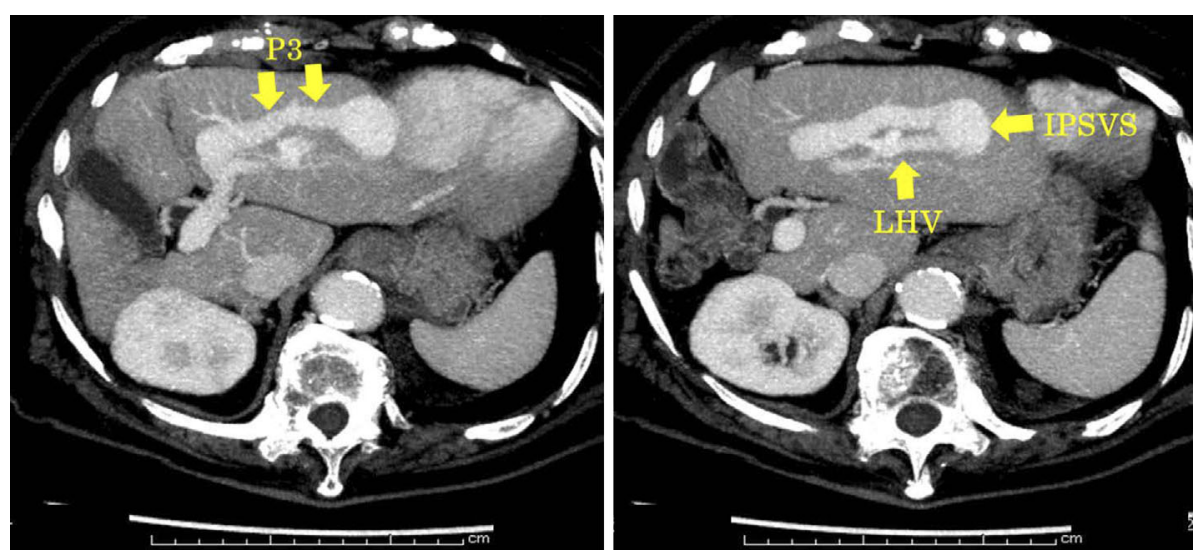

Figure 1. Images of an enhanced abdominal computed tomography scan showing the intrahepatic portosystemic venous shunt. IPSVS: intrahepatic portosystemic venous shunt, LHV: left hepatic vein, P3: lateral inferior branch of the portal vein

$3 / 5$ in both legs. The patellar and Achilles' tendon reflexes were increased bilaterally. The patient's muscle tone in both legs was moderately increased and her muscle strength, tone and tendon reflexes in the upper limbs were intact. The cranial nerve functions and sensory system were normal throughout the patient's entire body. The bladder and bowel functions were preserved. The laboratory data, including the serum electrolyte levels, liver function tests and a thyroid function test, showed no significant abnormalities (Table). The results of hepatitis B surface antigen and hepatitis $C$ antibody tests were both negative. Plain computed tomography (CT) and magnetic resonance imaging (MRI) of the brain detected old bilateral lacunar infarcts in the basal ganglia; however, no signs of acute cerebrovascular disease were observed. In consideration of the patient's acute clinical course, the presence of several atherosclerotic comorbidities and the absence of a clear-cut etiology, a type of TIA, particularly that occurring in the vertebrobasilar system, was strongly suspected. We administered intravenous ozagrel sodium (80 mg/day) for seven days. The patient's neurological symptoms almost completely disappeared within one day; however, on the seventh day of injection, spastic paraparesis with acute alteration of consciousness appeared. Additional diagnostic tests revealed hyperammonemia at a level of 192 $\mu \mathrm{g} / \mathrm{dL}$ (normal, 30-86 $\mu \mathrm{g} / \mathrm{dL}$ ) without distinct hepatic dysfunction. Even in retrospect, no areas of high signal intensity of globus pallidum on T1-weighted MRI, which is a characteristic finding of hepatic encephalopathy, were observed at the time of admission. To further investigate the condition underlying the hyperammonemia, we performed enhanced abdominal CT and detected an IPSVS. Because the patient's medical history, physical condition and other examination findings did not demonstrate any hepatic pathologies predisposing to secondary portosystemic shunt formation, the IPSVS was considered to be congenital.

The IPSVS measured $25 \mathrm{~mm}$ in diameter and was located 


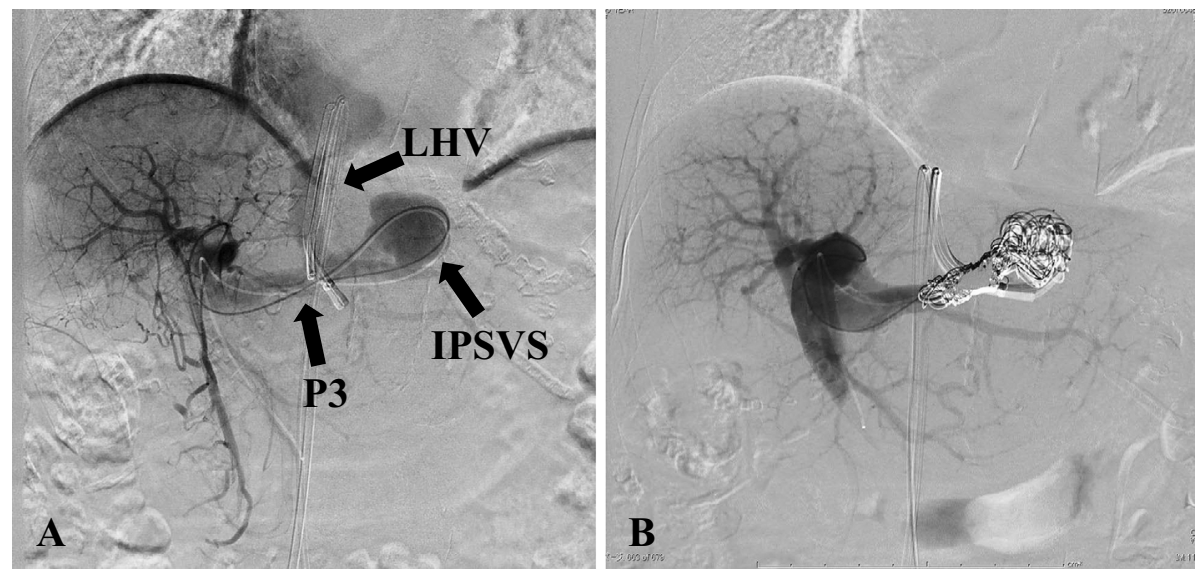

Figure 2. Images of an intrahepatic portosystemic venous shunt during transcaval retrograde portography. A. The large shunt is visualized prior to embolization. B. Embolic coils were placed in the shunt.

in the left lobe, communicating from the lateral inferior branch of the portal vein to the left hepatic vein (Fig. 1). Although the patient's consciousness was restored with branched-chain amino acids, lactulose and a low protein diet, her serum ammonia level remained high, and the paraparesis did not completely resolve, even after rehabilitation. We decided to perform transcatheter embolization for IPSVS. A retrograde transcaval approach via the right femoral vein was chosen to access the portal system, and metallic coils were used to embolize the shunt (Fig. 2). The portal pressure before embolization was a normal level of 100 $\mathrm{mmH}_{2} \mathrm{O}$. The portal pressure remained at the same level, and the serum ammonia level immediately decreased to a normal level after embolization. This treatment resulted in the improvement of the remaining slight paraparesis, and the patient completely regained her ability to walk. Her clinical status and serum ammonia level have stayed normal during a follow-up period of 20 months, even without medication or dietary therapy.

\section{Discussion}

IPSVS is an abnormal vessel that communicates from the portal vein to the hepatic vein. It often causes neurological symptoms characteristic of HE. Altered consciousness, such as impaired attention, confusion and disorientation, is typically the initial prominent symptom. The condition can progress to more irrational behavior, lethargy and finally coma $(1,2)$. Most cases of IPSVS usually occur following the development of portal hypertension due to chronic hepatic dysfunction, particularly hepatic cirrhosis $(3,4)$. Portal embolism and idiopathic portal hypertension are also known to cause portal hypertension. Hepatic surgery or trauma can also result in IPSVS during the healing process. The occurrence of IPSVS without these conditions or a related background is usually classified as congenital, similar to that observed in the present case (5). Congenital IPSVS is an uncommon disease, with a reported prevalence in the general adult population of $0.0235 \%$ (6). Recently, significant advances in imaging techniques have helped to increase reports of newly detected asymptomatic cases (5). The shunt size and flow rate are reported to increase in association with age (7), and the amount of shunt blood flow correlates with the onset of HE. A shunt ratio of $>30 \%$ is a risk factor for $\mathrm{HE}$, with that of $\geq 60 \%$ predisposing the patient to a high risk of HE (8). In addition, the tolerance of the central nervous system to neurotoxic substances, including ammonia, decreases as the patient becomes older (9). Accordingly, congenital IPSVS often presents with initial symptomatic onset at an age of $\geq 50$ years $(10,11)$.

In patients with IPSVS, ammonia and several other kinds of neurotoxins are produced in the gastrointestinal tract that directly reach the systemic circulation and exert harmful effects on the brain function. Astrocytes, which primarily compose and sustain the blood brain barrier, are involved in the homeostasis and nutrition of neurons. Ammonia-induced astrocyte swelling and dysfunction are known to be a critical part of the pathophysiology of HE. In patients with hyperammonemia, the detoxification process for ammonia results in the synthesis of excessive amounts of glutamine in astrocytes. The glutamine evokes oxidative/nitrosative stress and mitochondrial abnormalities in astrocytes, which lead to cell degeneration (12-14). Ammonia also accelerates the neuroinhibitory actions of gamma-aminobutyric acid and endogenous benzodiazepine, resulting in altered consciousness and psychomotor dysfunction $(2,15)$. Our patient initially presented with acutely progressive paraparesis without a distinct alteration in consciousness. After the initial examination, we strongly suspected that she had a cerebrovascular disease, considering all of her medical information on admission, specifically, the acute onset at an older age, past history of TIA and arteriosclerotic comorbidities of type 2 diabetes mellitus, dyslipidemia and hypertension. There are several reported cases of $\mathrm{HE}$ in which focal neurological deficits have occurred. The symptoms of these patients varied widely, e.g., ocular motor paralysis, hemiparesis, hemi- 
plegia and paraparesis have been reported (16-19). Cases of HE associated with paraparesis have been diagnosed as "hepatic myelopathy" because the myelopathic symptoms were clinically correlated with $\operatorname{HE}(2,20)$. Hepatic myelopathy typically presents as spastic paraparesis without obvious sensory involvement and is pathologically characterized by demyelination or the axonal loss of pyramidal tracts $(18,20,21)$. The lesions are located in the spinal cord and are often bilateral. However, no specific abnormalities are found on cerebral or spinal MRI in patients with hepatic myelopathy. In our patient, detailed examinations, including cerebral CT and MRI, detected no obvious causes of the symptoms, other than hyperammonemia, and the spastic paraparesis was consistent with hepatic myelopathy in that it worsened and improved in step with the status of HE. The improvement of paraparesis immediately after antiplatelet therapy is considered to be the result of the correction of dehydration and the diluted concentration of serum ammonia using Ringer's solution. The patient's normal mental status at the initial onset of hepatic myelopathy was actually an uncommon clinical course because HE typically precedes myelopathy. However, other similar uncommon cases have been reported $(4,18)$.

In conclusion, we herein reported a case of hepatic myelopathy due to congenital IPSVS. To our knowledge, such a case has not been previously reported in the English language literature. Diagnosing this disease was difficult because acute paraparesis with normal cerebral imaging studies is also observed in cases of vertebrobasilar TIA. In addition, the patient's old age, normal mental status, past history and atherosclerotic comorbidities suggested a diagnosis of TIA. However, her neurological symptoms persisted for several hours, which is uncharacteristically long for TIA (22). Diagnosing TIA always has a degree of subjectivity and uncertainty because the diagnosis is largely based on the patient's narrative and physical findings, and laboratory and imaging tests do not provide diagnostically definitive proof. Therefore, if paraparesis due to TIA is suspected and the symptoms, findings and/or therapeutic outcomes are not completely consistent with TIA, then a diagnosis of hepatic myelopathy due to congenital IPSVS should be ruled out.

The authors state that they have no Conflict of Interest (COI).

\section{References}

1. Blei AT, Cordoba J. Hepatic encephalopathy. Am J Gastroenterol 96: 1968-1976, 2001.

2. Lewis M, Howdle PD. The neurology of liver failure. QJM 96: 623-633, 2003.
3. Miyata K, Tamai H, Uno A, et al. Congenital portal systemic encephalopathy misdiagnosed as senile dementia. Intern Med 48: 321-324, 2009.

4. Wang MQ, Liu FY, Duan F. Management of surgical splenorenal shunt-related hepatic myelopathy with endovascular interventional techniques. World J Gastroenterol 18: 7104-7108, 2012.

5. Tanoue S, Kiyosue H, Komatsu E, Hori Y, Maeda T, Mori H. Symptomatic intrahepatic portosystemic venous shunt: embolization with an alternative approach. AJR Am J Roentgenol 181: 7178, 2003.

6. Lin ZY, Chen SC, Hsieh MY, Wang CW, Chuang WL, Wang LY. Incidence and clinical significance of spontaneous intrahepatic portosystemic venous shunts detected by sonography in adults without potential cause. J Clin Ultrasound 34: 22-26, 2006.

7. Kiriyama M, Takashima S, Sahara H, et al. Case report: portalsystemic encephalopathy due to a congenital extrahepatic portosystemic shunt. J Gastroenterol Hepatol 11: 626-629, 1996.

8. Uchino T, Matsuda I, Endo F. The long-term prognosis of congenital portosystemic venous shunt. J Pediatr 135: 254-256, 1999.

9. Raskin NH, Price JB, Fishman RA. Portal-systemic encephalopathy due to congenital intrahepatic shunts. N Engl J Med 270: 225229, 1964.

10. Oguz B, Akata D, Balkanci F, Akhan O. Intrahepatic portosystemic venous shunt: diagnosis by colour/power Doppler imaging and three-dimensional ultrasound. Br J Radiol 76: 487-490, 2003.

11. Park JH, Cha SH, Han JK, Han MC. Intrahepatic portosystemic venous shunt. AJR Am J Roentgenol 155: 527-528, 1990.

12. Schliess F, Gorg B, Haussinger D. RNA oxidation and zinc in hepatic encephalopathy and hyperammonemia. Metab Brain Dis 24: 119-134, 2009.

13. Riggio O, Ridola L, Pasquale C. Hepatic encephalopathy therapy: An overview. World J Gastrointest Pharmacol Ther 1: 54-63, 2010.

14. Albrecht J, Norenberg MD. Glutamine: a Trojan horse in ammonia neurotoxicity. Hepatology 44: 788-794, 2006.

15. Basile AS, Jones EA. Ammonia and GABA-ergic neurotransmission: interrelated factors in the pathogenesis of hepatic encephalopathy. Hepatology 25: 1303-1305, 1997.

16. Cadranel JF, Lebiez E, Di Martino V, et al. Focal neurological signs in hepatic encephalopathy in cirrhotic patients: an underestimated entity? Am J Gastroenterol 96: 515-518, 2001.

17. Averbuch-Heller L, Meiner $Z$. Reversible periodic alternating gaze deviation in hepatic encephalopathy. Neurology 45: 191-192, 1995.

18. Lewis MB, MacQuillan G, Bamford JM, Howdle PD. Delayed myelopathic presentation of the acquired hepatocerebral degeneration syndrome. Neurology 54: 1011, 2000.

19. Atchison JW, Pellegrino M, Herbers P, Tipton B, Matkovic V. Hepatic encephalopathy mimicking stroke. A case report. Am J Phys Med Rehabil 71: 114-118, 1992.

20. Sobukawa E, Sakimura K, Hoshino S, Hoshino M, Miyoshi K. Hepatic myelopathy: an unusual neurological complication of advanced hepatic disease. Intern Med 33: 718-722, 1994.

21. Zieve L, Mendelson DF, Goepfert M. Shunt encephalomyelopathy. II. Occurrence of permanent myelopathy. Ann Intern Med 53: 5363, 1960.

22. Sorensen AG, Ay H. Transient ischemic attack: definition, diagnosis, and risk stratification. Neuroimaging Clin N Am 21: 303-313, 2011.

(C) 2013 The Japanese Society of Internal Medicine http://www.naika.or.jp/imonline/index.html 